\title{
Precise shape measurements of rotating workpieces by a single optical sensor
}

\author{
R. Kuschmierz ${ }^{1}, P$. Günther ${ }^{1}$, J. Czarske ${ }^{1}$ \\ ${ }^{1}$ Laboratory of Measurement and Testing Techniques, TU Dresden, 01062 Dresden \\ juergen.czarske@tu-dresden.de
}

\begin{abstract}
:
The precise measurement of the shape of fast rotating workpieces is important at several applications such as lathe monitoring. For such tasks the laser Doppler distance sensor technique was invented by the TU Dresden some years ago. This technique has been realized by two mutually tilted interference fringe systems, where the distance is coded in the phase difference between the generated interference signals of two photo detectors. However, due to the speckle effect at rough surfaces, random envelopes and phase jumps occur disturbing the phase difference estimation. Recently a scientific breakthrough for the measurement uncertainty budget has been achieved. Via illumination and receiving optics matching the uncertainty of the measurement uncertainty was reduced by about one magnitude. For displacement measurements of a recurring rough surface an uncertainty of 110 $\mathrm{nm}$ were attained. It enables precise shape measurements, since the lateral velocity and the rotational speed of rotating objects are determined additionally. This novel sensor technique can be advantageously used for the quality control of workpieces inside of a lathe towards the reduction of process tolerances, installation times and costs.
\end{abstract}

Key words: interferometry, phase measurement, speckle, process monitoring and control, rough surface, sensor data fusion, multimodal measurements.

\section{Introduction}

The precise measurement of the vibration, diameter and shape of fast rotating workpieces is important at several applications. One task is the monitoring of the workpiece diameter inside of a lathe. The tolerances of the workpiece dimensions are reduced more and more. Currently distance measurement techniques often have to offer uncertainties in the submicron range. Optical sensors such as triangulation [1], low coherence tomography [2] or digital holographic interferometry [3] can fulfill this demand. However, by using these distance measurement techniques, two sensors have to be employed in order to determine the workpiece diameter, see Fig. 1 (left). The distance $A$ between the sensors has to be known a priori with submicron precision. Due to temperature changes and vibrations, which usually are occurring in lathes, it is very difficult to guarantee this precision during the measurements.

In order to overcome this drawback the laser Doppler distance sensor with phase coding ( $P$ LDDS) was invented. The novel interferometer setup based on two mutually tilted interference fringe systems enables the determination of the diameter of the workpiece with only one single sensor by measuring the axial distance and the lateral velocity of the rotating workpiece simultaneously, see Fig. 1 (right).

\section{Sensor principle}

Laser Doppler velocimeters (LDV) are based on the evaluation of scattered light signals which are generated from measurement objects passing the interference fringe system in the intersection volume of two coherent laser beams. These scattered light signals exhibit an amplitude modulation with the Doppler frequency $f$. Thus, the measurement object velocity $v$ can be calculated by [4]:

$v=f \cdot d$,

where $d$ is the mean fringe spacing due to the sensor setup. In order to obtain the z-position of the measurement object simultaneously, an extended LDV setup with two slightly tilted interference fringe systems and a phase evaluation of the two scattered light signals is used, see Fig. 2. 

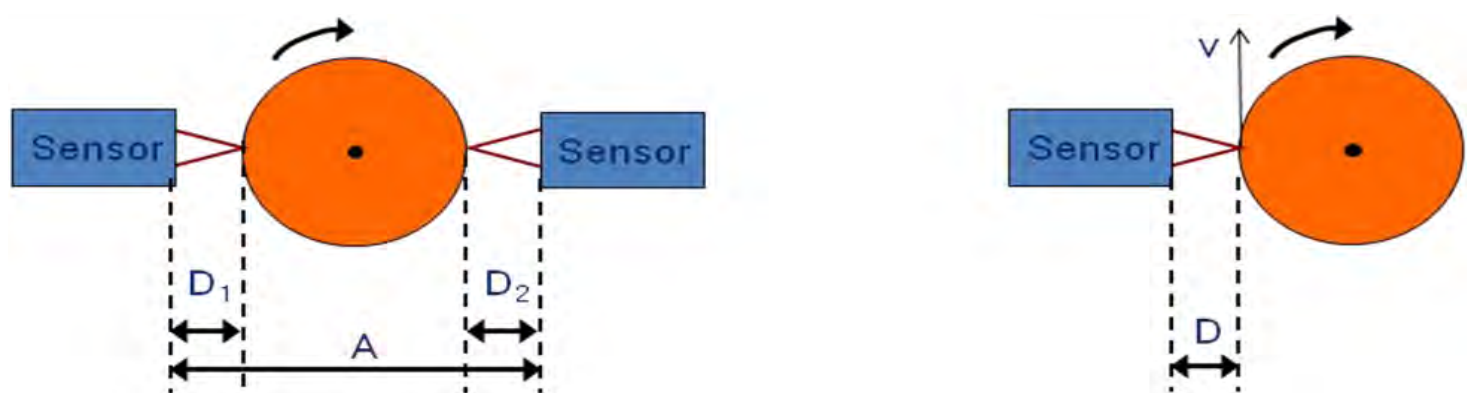

Fig. 1: Two-dimensional shape determination of rotating objects by the measurement of the angle resolved diameter.

Left: Conventional method based on two distance sensors. The diameter of the object is determined by the sensors clearance $A$ and the two distance measurements $D_{1}$ and $D_{2}$. The sensor clearance $A$ is subject of temperature and vibration influences.

Right: Novel method based on the simultaneous measurement of lateral velocity $v$ and distance $D$. The diameter and the shape are determined by only one single sensor. Vibration and temperature influences are not critical, since they are measured by the sensor.

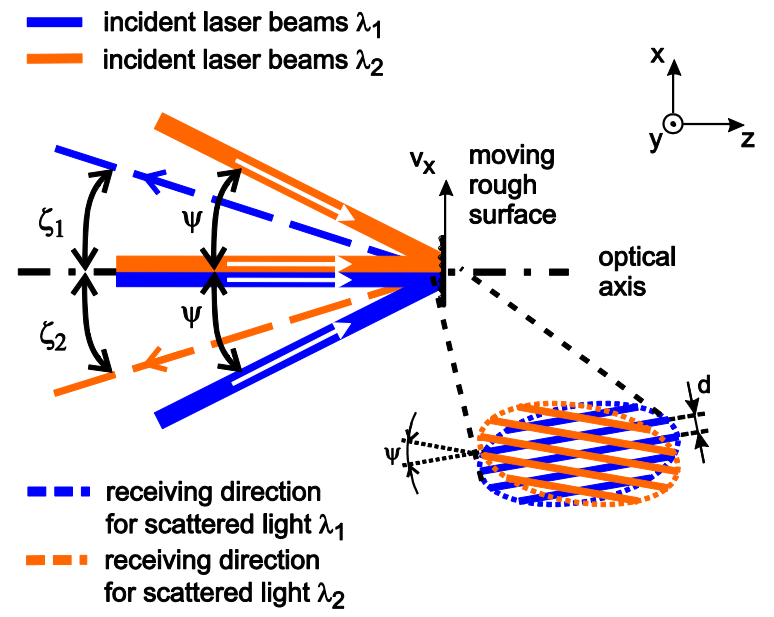

Fig. 2: Principle of the laser Doppler distance sensor with phase coding. Superposition of two interference fringe systems with equal fringe spacing $d$, which are tilted by an angle $\psi[5]$.

When a scattering object crosses this measurement volume, two distinguishable scattered light signals result. These two signals exhibit a phase difference $\varphi$ depending on the axial position $z$ of the scattering object. Assuming plane wavefronts, this phase difference can be described as:

$\varphi=s \cdot z+\varphi_{0}$,

where $s$ is the slope of the phase difference function $\varphi(z)$ and $\varphi_{0}$ the phase offset in the center of the measurement volume $(z=0)$. By evaluating the phase difference, the position $z$ inside the measurement volume can be determined using the inverse function of Eq. (2). With the known working distance $D_{0}$ between sensor front face and the center of the measurement volume, also the distance
$D=D_{0}+z$ of the measurement object with respect to the sensor can be determined.

In order to achieve a low distance measurement uncertainty of about one micron, a steep phase function $\varphi(z)$, i. e. a large tilting angle $\psi$, is necessary due to [6] :

$\sigma_{z}=S^{-1} \sigma_{\varphi}$.

However, the similarity of the two scattered light signals from a rough surface decreases with increasing tilting angle $\psi$, if both signals are detected from one receiving direction $\left(\zeta_{1}=\zeta_{2}=\right.$ $0^{\circ}$ ), see Fig. 3, where interference signals of rough surfaces were simulated using MATLAB in order to study the dependency. The crosscorrelation coefficient $\rho$ was calculated as a value for the similarity of the two mean-free scattered light signals.

The simulations show that the correlation coefficient is reduced significantly by increasing the tilting angle $\psi$. A further reduction of similarity is caused by using different wavelengths for channel discrimination. This is due to the speckle effect at rough surfaces, which depends on the laser wavelength as well as on the angle of the incident light. With increasing tilting angle $\psi$ and wavelength difference $\Delta \lambda$ the speckle pattern of both interference fringe systems becomes more unsimilar resulting in different envelopes and phase jumps at different times. As further shown in Fig. 4, the decrease of the correlation of the two measurement signals results in a strong increase of the measurement uncertainty of the phase difference $\sigma_{\varphi}$ and, thus, in a strong increase of the distance measurement uncertainty, compare Eq. (3). 


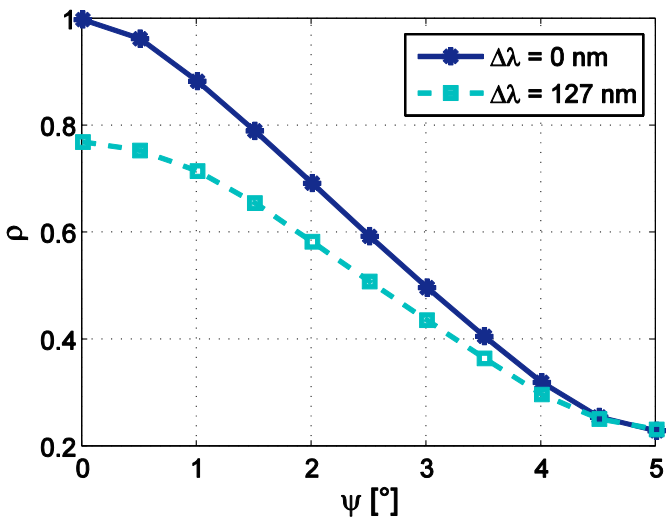

Fig. 3: Simulation: Correlation coefficient $\rho$ of the two scattered light signals for different tilting angles $\psi$. Noise sources are not considered [6].

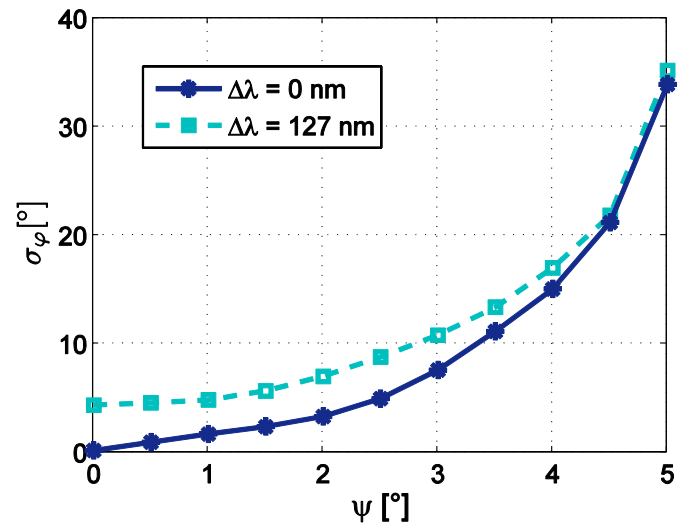

Fig. 4: Simulation: Phase difference uncertainty $\sigma_{\varphi}$ for different tilting angles $\psi$. Noise sources are not considered [5].

However, the speckle effect depends not only on the incidence angle of the laser light. Also the receiving angle of the light detection unit has an influence. In order to evaluate this effect, the correlation coefficient $\rho$ from two scattered light signals from a rough surface was determined for different receiving angles $\zeta_{1}$ and $\zeta_{2}$, compare Fig. 2 . As it can be seen clearly in Fig. 5, the correlation coefficient depends strongly on these receiving angles. For optimum receiving angle, a correlation coefficient of about 0.8 was achieved, whereas the correlation coefficient decreases to 0.2 when the receiving angles were suboptimal. The measurements show, that the maximum correlation coefficient was achieved when the sum of the two receiving angles $\zeta_{1}$ and $\zeta_{2}$ is equal to the tilting angle $\psi$ of the two interference fringe systems: $\psi=\zeta_{1}+\zeta_{2}$. Regarding the simulations in Figures 3 and 4 , the uncertainty of the phase difference $\sigma_{\varphi}$ can be decreased by the optimization the detection optics from $\sigma_{\varphi}=35^{\circ}$ at $\rho=0.2$ to $\sigma_{\varphi}=4^{\circ}$ at $\rho=$ 0.8 .

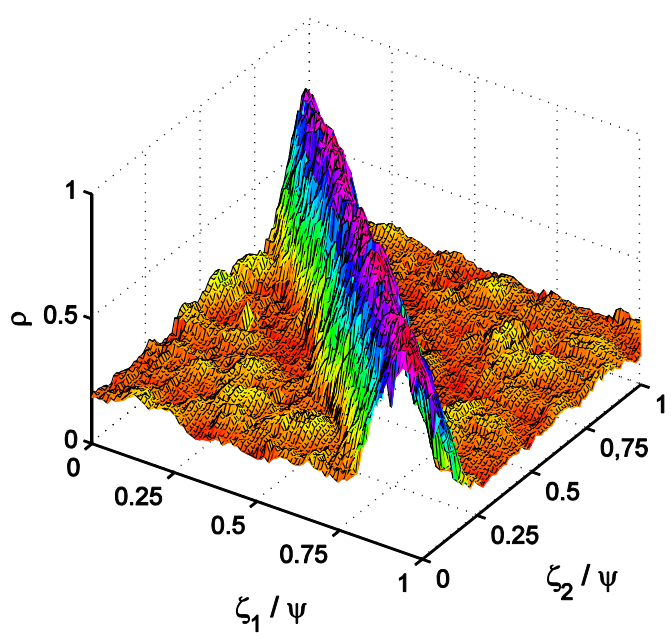

Fig. 5: Experiment: (a) Correlation coefficient in dependence on the angle $\left(\zeta_{1}+\zeta_{2}\right)$ between the two receiving angles in relation to the tilting angle $\psi$ between the interference fringe systems. The correlation coefficient reach the maximum at $\psi=\zeta_{1}+$ $\zeta_{2}$

\section{Experimental setup}

Due to demands previously found out and disccussed in the previous section, the sensor was designed as shown in Fig. 5. Two red laser diodes $\left(\lambda_{1}=658 \mathrm{~nm}, \lambda_{2}=685 \mathrm{~nm}\right)$ exhibiting a maximum power of about $20 \mathrm{~mW}$ were used as light sources. Hereby, the small wavelength difference is of benefit to minimize the wavelength influence on the similarity of the two interference signals. The two laser beams were focused on a transmission phase grating, which acts as beam splitter. The first positive and negative diffraction orders were used as partial beams, all other orders were blocked by beam stops. A Keplerian telescope focused the partial beams forming the measurement volume with the two tilted interference fringe systems with a length in z-direction, i. e. the distance measurement range of $200 \mu \mathrm{m}$.

According to Eq. (3), a high slope of the phase function $\varphi(z)$ is demanded. Therefore, a small fringe spacing of $d=3 \mu \mathrm{m}$ and a high tilting angle $\psi=7.5^{\circ}$ between the two interference fringe systems were realized. The tilting angle $\psi$ is adjusted by shifting the mirror in front of the grating. Furthermore the laser beam $\lambda_{2}$ is shifted in $x$-direction, see the arrows in Fig. 6 . The slope $s$ results in $12.9 \%$ m corresponding to an increment length of the phase function $\varphi(\mathrm{z})$ of $\mathrm{I}_{2 \pi}=28 \mu \mathrm{m}$. 


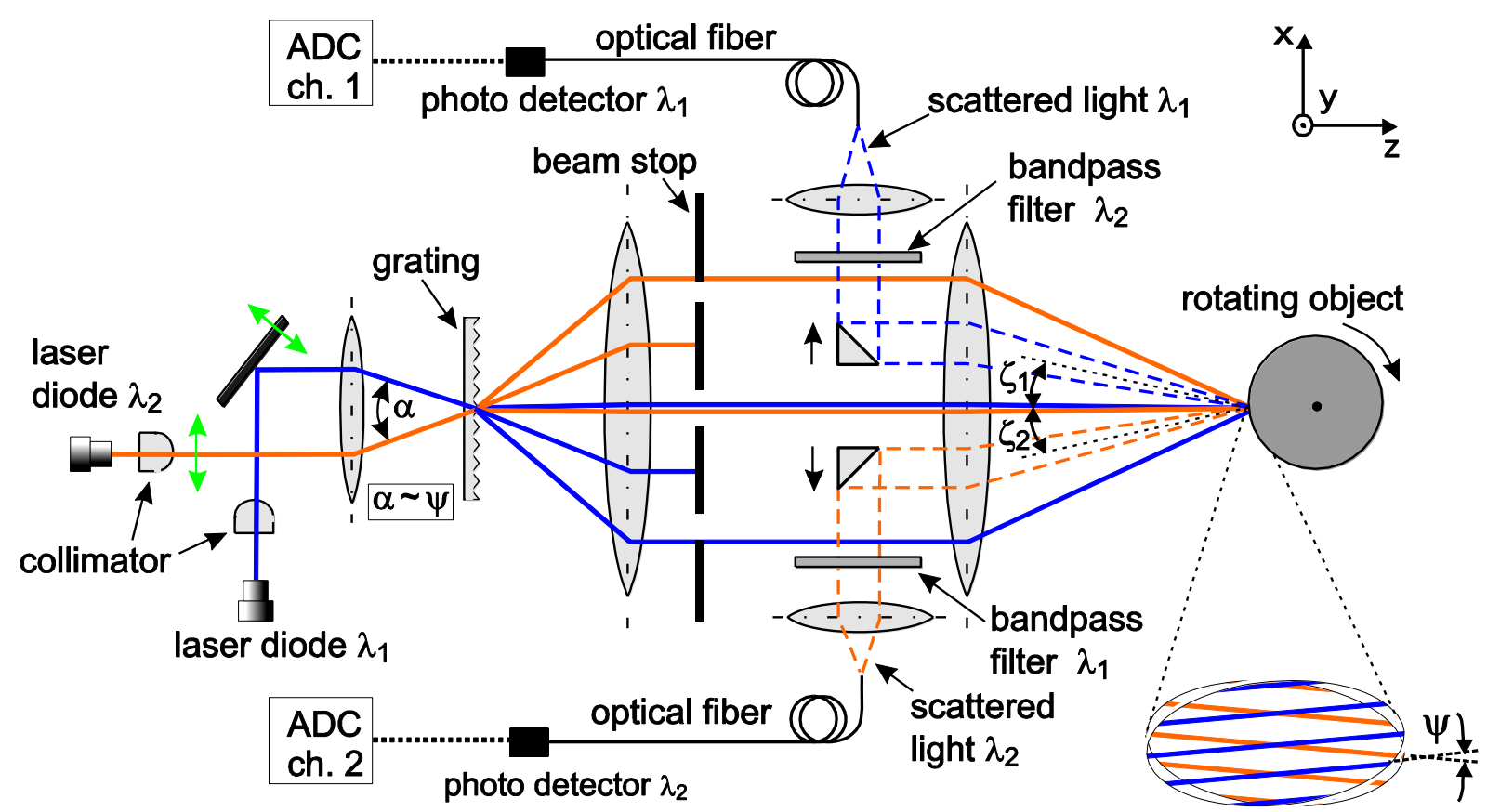

Fig. 6: Setup of the laser Doppler distance sensor with tilted interference fringe systems and receiving optic matching with $\psi=\zeta_{1}+\zeta_{2}$.

The bi-chromatic scattered light from the measurement object was detected in backward direction. Collimated by the front lens, the scattered light was coupled out symmetrically by two small mirrors between the partial beams inside the Keplerian telescope, see Fig. 6. In order to achieve a high correlation coefficient of both interference signals, a separate receiving direction is utilized for each laser wavelength. The other laser wavelength is blocked by interference bandpass filters, respectively. Afterwards, the two monochromatic scattered light signals are coupled into multimode fiber patch cables (400 $\mu \mathrm{m}$ diameter) and guided to photo detectors. The electrical photo detector signals were sampled simultaneously by a 12bit $A / D$ converter card $(A D C)$ installed in a standard PC.

The digital signal processing and evaluation was done using MATLAB. The Doppler frequencies $f_{D 1}, f_{D 2}$ were calculated with a least square regression of the fast Fourier transformed photo detector signals. For phase estimation the cross-correlation function of the two photo detector signals was calculated. Via a cosine least square fit, the time shift $\Delta t$ of the maximum of the cross-correlation function was determined, which is proportional to the phase difference $\varphi=2 \pi f_{D} \Delta t[6]$.

\section{Distance measurement}

In order to demonstrate the influence of the signal similarity on the distance measurement uncertainty experimentally, the distance of a tumbling brass disc exhibiting at a lateral velocity of about $10 \mathrm{~m} / \mathrm{s}$ was measured, see Fig. 7 (left). The tumbling of the disc results in a sinusoidal distance variation to the P-LDDS. At first, the measurement was accomplished with a mismatched setup of the receiving optic resulting in a low correlation coefficient of only $\rho=0.4$. Additionally, a second measurement with matched receiving optic $\left(\psi=\zeta_{1}+\zeta_{2}\right)$ features a high correlation coefficient of $\rho=0.7$. The measured distance variation $z$ for both setups is depicted in Fig. 7 (right). The significantly lower measurement uncertainty of the well adjusted sensor setup is clearly visible. The mismatched setup results in a standard deviation of $2.2 \mu \mathrm{m}$ and a maximum deviation of the mean value of about $8 \mu \mathrm{m}$ whereas the matched setup exhibit a standard deviation of $0.8 \mu \mathrm{m}$ and a maximum deviation of the mean value of only $1.5 \mu \mathrm{m}$. Thus, the overall measurement uncertainty

$\sigma_{z, t o t}=\sqrt{\sigma_{z}^{2}+\frac{|\Delta z|^{2}}{3}}$

is reduced significantly from $\sigma_{z \text {,tot }}=5.1 \mu \mathrm{m}$ to $\sigma_{z, \text { tot }}=1.2 \mu \mathrm{m}$ via the receiving optic matching. 

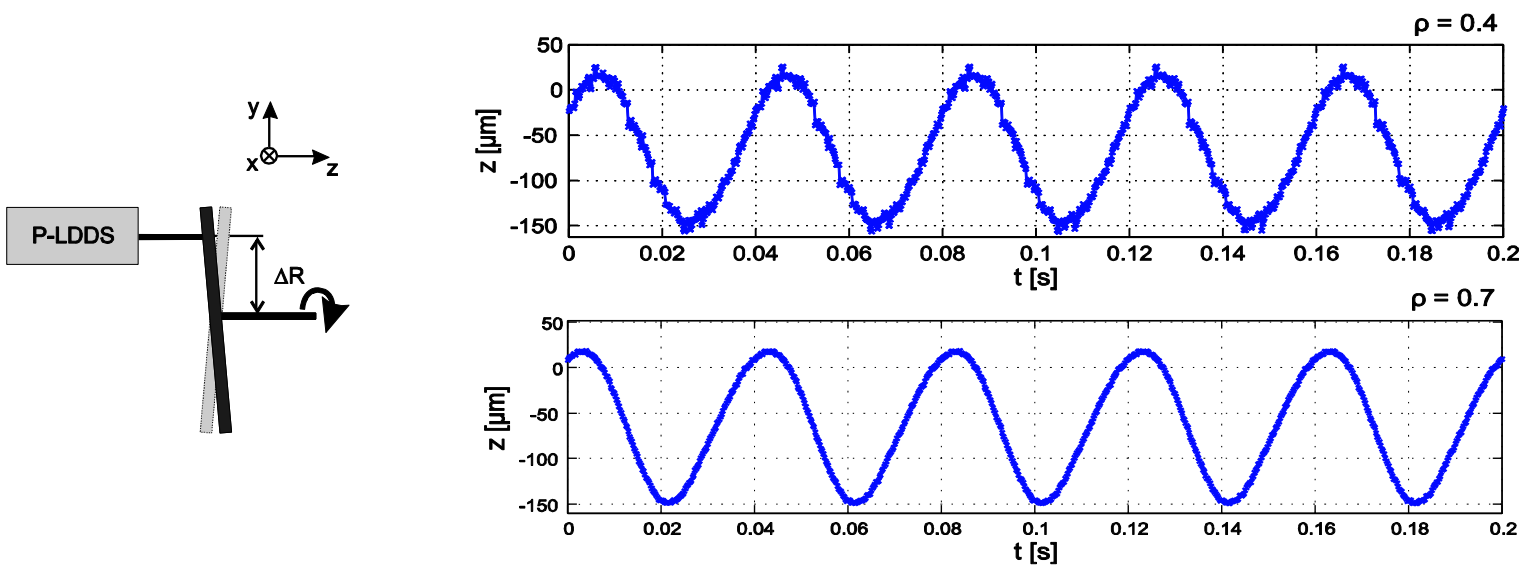

Fig. 7: Measurement setup (left) and measured distance variation with misadjusted (right top) and well adjusted receiving optic of the P-LDDS (right bottom) [5].

\section{Shape measurement}

Due to the fact, that the P-LDDS enables the determination of the distance and the velocity simultaneously, the absolute two-dimensional shape of rotating objects can be measured. Therefore, no a-priori knowledge of the distance between sensor and rotation center of the measurement object is necessary. From the mean surface velocity $\langle v\rangle$ and the mean rotational speed $\left\langle f_{\text {rot }}\right\rangle$, the mean absolute radius $\langle R>$ was calculated by [8]

$$
\langle R\rangle=\frac{\langle v\rangle}{2 \pi\left\langle f_{\text {rot }}\right\rangle}
$$

Thereby, the rotational speed $f_{\text {rot }}$ can be determined by the correlation analysis of the scattered time signals. The time-resolved angular speed $\omega(t)$ of the rotating object is given by

$\omega(t)=\frac{v(t)}{\langle R\rangle+\Delta r(t)}$,

where $\Delta r(t)=z(t)-\langle z\rangle$ is the mean-free distance variation. By integrating the angular speed over time, the rotation angle $\phi(t)$ was determined. Thus, the time-resolved shape of a rotating object can be determined by:

$$
R(t)=\left(\begin{array}{l}
x \\
y
\end{array}\right)=\left[\langle R\rangle+\Delta r(t)\left(\begin{array}{c}
\cos [\phi(t)] \\
\sin [\phi(t)]
\end{array}\right)\right. \text {. }
$$

In Fig. 8, the two-dimensional shape of the rotating cylinder measured with the P-LDDS is depicted, which is in this case a decentered circle. The distance information $z(t)$ is zoomed by the factor of 50 in order to see the eccentric clamping of the cylinder. The measurement uncertainty of the absolute radius $R(t)$ can be calculated using the law of propagation of uncertainty. Thus, the standard uncertainty of the radius is $\sigma_{R}=\sqrt{\sigma_{\langle R\rangle}^{2}+\sigma_{z, t o t}^{2}}$.

Taking into account the relative velocity uncertainty $\sigma_{v x} / v_{x}=8 \cdot 10^{-4}$, the number of measurement points per revolution $\mathrm{N}=100$ and the mean radius $\angle R>=20 \mathrm{~mm}$ the uncertainty of the mean radius can be estimated with $\sigma_{\langle R\rangle}=\sigma_{v x}\langle R\rangle /\left(\sqrt{N} v_{x}\right)=1.6 \mu \mathrm{m}, \quad$ since uncorrelated consecutive values can be assumed. The standard uncertainty of the radius results in $\sigma_{\mathrm{R}}=1.8 \mu \mathrm{m}$. In conclusion, the shape and the rotational axis of the cylinder were determined with micron uncertainty and with only one sensor.

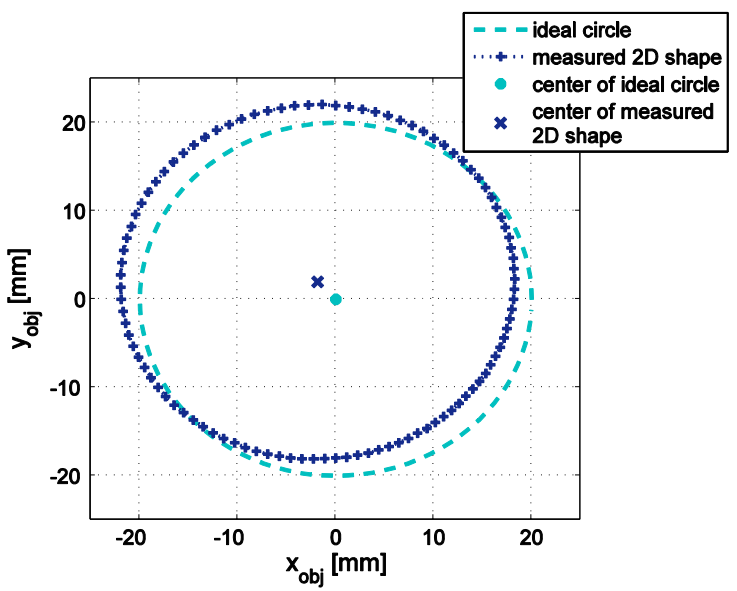

Fig. 8: Measured two-dimensional shape of the rotating aluminum cylinder in comparison with an ideal circle. The eccentricity of about $55 \mu \mathrm{m}$ determined by the distance information of the $P$ LDDS is zoomed by the factor of 50 . 


\section{Conclusion}

In this paper we have presented a setup of the laser Doppler distance sensor with phase difference estimation of two interference signals. The receiving optic matches the illumination optic. The measurement uncertainty of the phase difference $\varphi$ reduces significantly due to the enhancement of the similarity of the two interference signals by the matched receiving optic. The total distance measurement uncertainty is independent of the transvers velocity in good approximation $[6,7]$. Thus, it is particularly suitable for measuring fast moving objects for example at high speed turning and grinding processes as well as of camshafts and crankshafts. Due to the simultaneous distance and velocity measurement, the time-resolved absolute radius and the shape of rotating objects is determined with a single sensor. The velocity information enables to evaluate the tangential velocity fluctuation and the rotational vibration of the blades. With the distance information the radial vibration of a rotor can be determined simultaneously and independently. Thus, two-dimensional vibrations schemes can be studied with only one single sensor.

\section{References}

[1] R. G. Dorsch, G. Häusler, and J.M. Herrmann. Laser triangulation: fundamental uncertainty in distance measurement. Appl. Opt., 33:13061314, 1994.

[2] A. Kempe, S. Schlamp, and T. Rösgen. Lowcoherence interferometric tip-clearence probe. Optics Letters, 28:1323-1325, 2003.

[3] G. Pedrini, W. Osten, and M. E. Gusev. Highspeed digital holographic interferometry for vibration measurement. Appl. Opt., 45(15):34563462, May 2006.

[4] H.-E. Albrecht, M. Borys, N. Damaschke, and C. Tropea. Laser Doppler and Phase Doppler Measurement Techniques. Springer Verlag, Berlin, 2003.

[5] P. Günther, R. Kuschmierz, T. Pfister, and J. Czarske. Distance measurement technique using tilted interference fringe systems and receiving optic matching. Opt. Lett., 37(22):47024704, 2012.

[6] P. Günther, T. Pfister, L. Büttner, and J. Czarske. Laser Doppler distance sensor using phase evaluation. Opt. Express, 17(4):2611-2622, 2009.

[7] T. Pfister, L. Büttner, and J. Czarske. Laser Doppler profile sensor with sub-micrometre position resolution for velocity and absolute radius measurements of rotating objects. Meas. Sci. Technol., 16:627-641, 2005.
[8] F. Dreier, P. Günther, T. Pfister, and Czarske J. Miniaturized non-incremental interferometric fiber-optic distance sensor for turning process monitoring. Opt. Eng., 51:014402, 2012. 